\title{
A Compact Two-armed Slave Manipulator for Minimally Invasive Surgery of the Throat
}

\author{
Wei Wei, Kai Xu, Nabil Simaan \\ Advanced Robotics and Mechanism Applications (ARMA) laboratory \\ Columbia University \\ 500 West 120th street, New York, NY 10027 \\ ww2161@columbia.edu,kx2102@columbia.edu,ns2236@columbia.edu
}

\begin{abstract}
This paper presents the design, simulation and evaluation of a two-armed compact slave robot being designed for Minimally Invasive Surgery of the throat. This robot has two robotic surgical arms equipped with novel distal dexterity units that use novel miniature snake-like devices. The hybrid kinematic structure of this slave robot includes closed and serial kinematic chains and snake-like devices. Jacobian formulation and redundancy resolution are presented together with a suturing simulation that evaluates the joint motions for a circular suture. The results demonstrate the capability of this robot to perform suturing inside a deep and confined surgical field such as the throat.
\end{abstract}

Index Terms - Dexterity, minimally invasive, throat, parallel robot, snake robots.

\section{INTRODUCTION}

In Minimally Invasive Surgery (MIS), e.g. laparoscopy, several tools are used to access the internal organs of the patient through three to five incisions in the abdomen. This limits the Degrees of Freedom (DoF) of all minimally invasive surgical tools to four DoF and sacrifices the dexterity of the surgical tools for the benefit of shortening the recovery time and reducing the patient's pain and the cost of surgery. While minimally invasive surgery proved successful in the last twenty years, it still faces several limitations. The biggest limitation is the lack of tool tip dexterity [1]. For these reasons many works focused on developing robotic assistants for MIS with distal dexterity enhancement devices that included planar and spatial linkages [2, 3], parallel wrists [4-6], serial articulated wrists [7-9], and more recently snake-like devices [9-16] (see [17], [18] for a comprehensive review of these systems and applications).

More recently, researchers have been developing robotic systems for Less Invasive Surgery (LIS) in which more than one surgical arm operate through a single access port. Ear, Nose, and Throat (ENT) surgery are examples where this approach is necessary, e.g. [19],[20]. This paper reports our recent efforts to develop a system for MIS of the throat and upper airways. While our previous works [21-23] focused on developing novel Distal Dexterity Units (DDU) to enable complex tissue operations in confined spaces, this paper focuses on the design of a complete two-armed slave. This novel two-armed slave has a hybrid kinematic structure that implements parallel and serial kinematic chains (including snake-like robots with actuation redundancy). The following section presents the layout of our robotic assistant. Then we present the kinematic modeling and Jacobian formulation of this novel robotic assistant and we perform simulations to demonstrate its capability to perform suturing inside very confined space.

\section{SYSTEM STRUCTURE}

\section{A. System Description}

The tele-robotic assistant for MIS of the upper airways is shown in Figs. 1, 2. This tele-robotic assistant consists of two Robotic Surgical Arms (RSA's). Both RSA's pass through a laryngoscope that provides access to the patient's upper airways, Fig. 2. The upper (proximal) end of each RSA is connected to a 2 DoF " $\mathrm{Z}-\theta$ stage" capable of rotating and translating the RSA about/along its own axis. Each Z- $\theta$ stage is connected to a 2 DoF five-bar manipulator via a passive universal joint. The distal end of each RSA passes through a supporting seal mounted inside the laryngoscope that in turn is affixed to the patient's bed and to the base of the tele-robotic assistant. The supporting seal serves as a fulcrum point inside the laryngoscope and eliminates the lateral deflections of the thin and long shafts of the RSA's. The pose of the tele-robotic slave manipulator is meant to mimic the current setup in manual surgery, The RSA's are connected to the Z- $\theta$ stages through fast-locking mechanisms that provide surgeons with a fast switch into manual surgery mode at any point they wish to do so (mainly in cases of emergency).

The distal end of each RSA is equipped with a $5 \mathrm{DoF}$ DDU, Fig. 3. The two DDU's serve as enabling components to compensate for the lost dexterity due to the tight constraints imposed by the small-diameter laryngoscope (a tube that is inserted into the patient's mouth to provide a clear pathway for surgical tools). They have been designed specifically to enable suturing inside the throat - an operation currently virtually impossible to operate in a manual minimally invasive fashion, [20, 21]. The DDU is located at the tip of the snake arm and composed of a snake-like unit (SLU) and a parallel manipulation unit (PMU). The SLU provides the necessary 


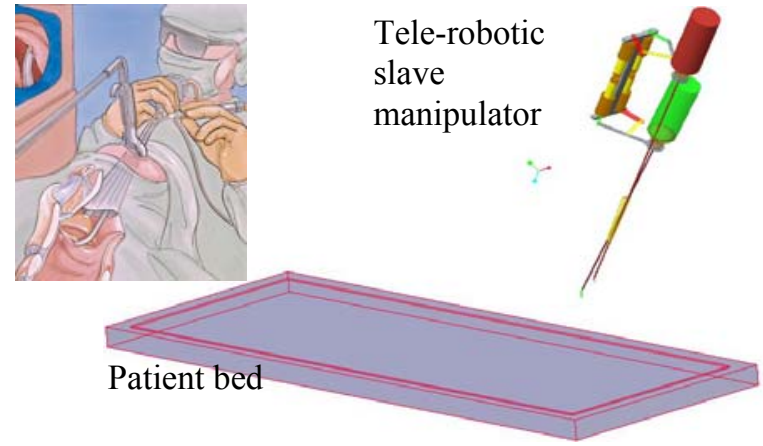

Figure 1. The tele-robotic two-armed manipulator is designed to be lightweight, compact and to preserve the current layout in manual surgery

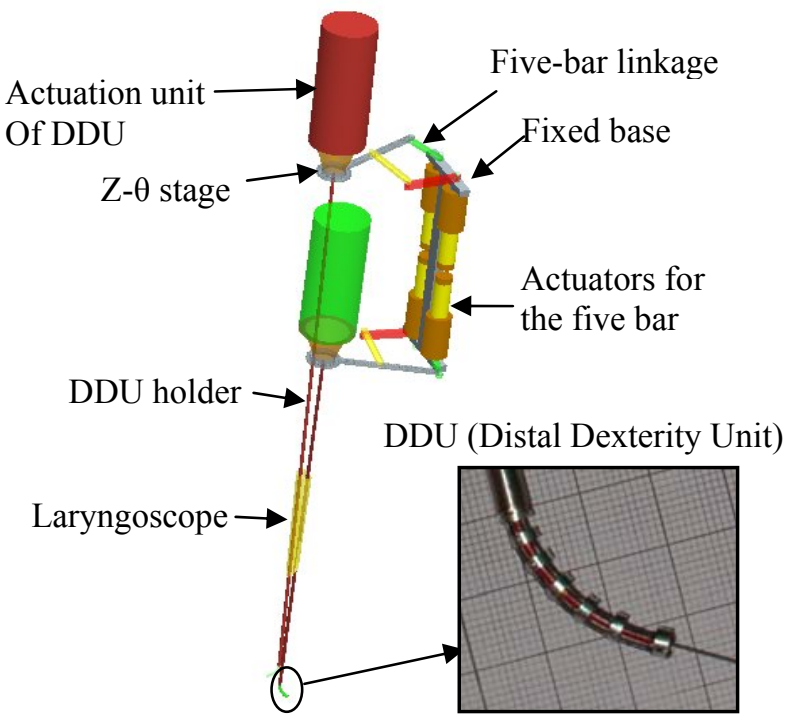

Figure 2. A detail of the two-armed tele-robotic assistant

motion capabilities inside the throat, while the PMU provides localized three DoF motion at the tip of the DDU with high accuracy. The SLU has four super-elastic NiTi tubes as its backbones. One primary backbone is located at the center and is attached to all the disks of the SLU. Three identical secondary backbones are equidistant from each other and from the primary backbone. The secondary backbones are only attached to the end disk and can slide through holes in the base and spacer disks. The PMU is located at the tip of the SLU and is actuated using the same methodology as well. Inside the backbones of the SLU, there are four more superelastic wires that actuate the PMU. There is also an additional wire passing through the central backbone to actuate the detachable gripper affixed to the moving platform of the PMU. The four interior wires can slide freely along the exterior ones, and actuators for all seven tubes are located in the actuation unit at the upper end of the snake-like robot.

\section{B. Design Parameters}

In the aim of assisting medical operations, the whole system needs to be designed out of minimized dimensions and weights. For example, the rotary actuated five-bar linkage is selected versus its linear actuated counterpart because with identical performances of the snake arm, rotary actuators save quite a lot of space and weights, which is a significant issue for surgeons. In addition, in this design, an optimization function is employed to minimize the required actuating torques of the five-bar linkage, as given in Eq. (1), where $\tau_{1}$ and $\tau_{2}$ are torques from the actuators. The five-bar linkage dimensions are determined so that the optimal one is actuated with minimum required torques.

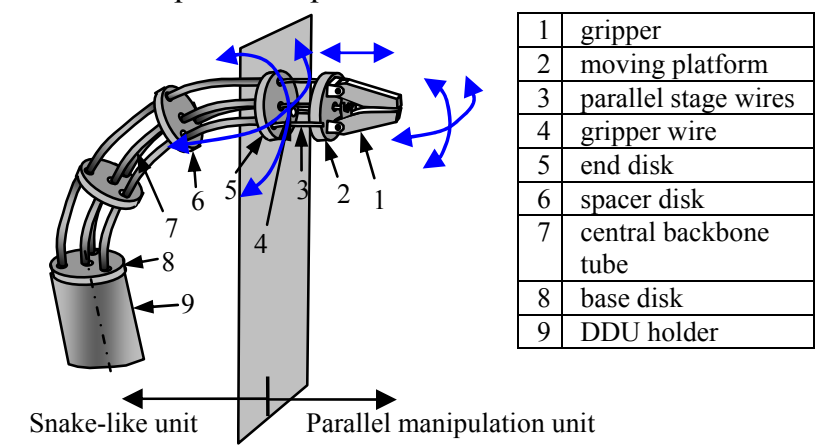

Figure 3. The Distal Dexterity Unit (DDU) composed from a multibackbone snake-like robot equipped with a parallel tip.

$$
\min f=\sqrt{\tau_{1}^{2}+\tau_{2}^{2}}
$$

Figure 4 shows the relative positions of the five-bar links. Two actuators control the angles of link 1 and link 2. The joint variables of these actuators are termed $q_{1}$ and $q_{2}$. The dimensional synthesis of this five bar was performed to allow its end effector to reach every point within a $60 \times 30 \mathrm{~mm}$ rectangular workspace. This required workspace was calculated based on the dimensions of the Karl Storz 8590 CV laryngoscope. Table I specifies the results of this dimensional synthesis.

The tip of the DDU's of the assembled two-armed slave robot is required to reach every point in a cylindrical workspace $50 \mathrm{~mm}$ in diameter and $100 \mathrm{~mm}$ in height. This workspace represents the surgical field of the larynx [20]. The required positional accuracy is better than $0.1 \mathrm{~mm}$, and the orientation precision is better than $1 / 3$ degree. The lengths of the snake arms are $550 \mathrm{~mm}$ and $800 \mathrm{~mm}$ for the two RSA's. The outer diameter of the tubes carrying the DDU (called DDU holders, Fig. 2) is $4.8 \mathrm{~mm}$.

$$
\text { TABLE I }
$$

OPTIMAL LINK DIMENSIONS

\begin{tabular}{|c|c|c|c|c|c|}
\hline Link 1 & Link 2 & Link 3 & Link 4 & Link 5 & Link 6 \\
\hline $60 \mathrm{~mm}$ & $60 \mathrm{~mm}$ & $110 \mathrm{~mm}$ & $90 \mathrm{~mm}$ & $80 \mathrm{~mm}$ & $180 \mathrm{~mm}$ \\
\hline
\end{tabular}

\section{KINEMATIC MODELING}

This section presents the kinematics and the Jacobian formulation of the whole robotic system. Part of the result has been presented in [20] for the DDU. In this paper, the kinematics problem is solved by dividing each branch of the two-armed slave manipulator of Fig. 2 into four parts, namely five-bar linkage, Z- $\theta$ stage, SLU, and PMU. The kinematics and Jacobian matrix for the whole system are obtained by integrating these four units together. The PMU is not fully analysed in detail in this paper. It is a subject of a separate future study. Instead, we consider the option of 
replacing the PMU with an additional snake-like manipulator. We will still refer to this unit as a PMU to maintain our terminology in Fig. 2. This arrangement reduces the DoF of the DDU from 5 to 4 (2 for each SLU).

\section{A. Kinematic Nomenclature}

Figure 4 shows a single robotic arm in a configuration in which the SLU and the PMU are bent in two different planes. The DDU is enlarged so that a clearer view can be acquired. In the system, nine coordinate systems are defined:

$\left\{\hat{\mathbf{x}}_{w}, \hat{\mathbf{y}}_{w}, \hat{\mathbf{z}}_{w}\right\}$ - World coordinate system (WCS)

$\left\{\hat{\mathbf{x}}_{t}, \hat{\mathbf{y}}_{t}, \hat{\mathbf{z}}_{t}\right\} \quad$ - Five-bar EE coordinate system (FES)

$\left\{\hat{\mathbf{x}}_{d}, \hat{\mathbf{y}}_{d}, \hat{\mathbf{z}}_{d}\right\}$ - Snake arm coordinate system (SAS)

$\left\{\hat{\mathbf{x}}_{b}, \hat{\mathbf{y}}_{b}, \hat{\mathbf{z}}_{b}\right\}$ - Base disk coordinate system (BDS)

$\left\{\hat{\mathbf{x}}_{1}, \hat{\mathbf{y}}_{1}, \hat{\mathbf{z}}_{1}\right\} \quad$ - Snake plane coordinate system (SPS)

$\left\{\hat{\mathbf{x}}_{e}, \hat{\mathbf{y}}_{e}, \hat{\mathbf{z}}_{e}\right\}$ - End disk coordinate system (EDS)

$\left\{\hat{\mathbf{x}}_{g}, \hat{\mathbf{y}}_{g}, \hat{\mathbf{z}}_{g}\right\}$ - Gripper base coordinate system (GCS)

$\left\{\hat{\mathbf{x}}_{2}, \hat{\mathbf{y}}_{2}, \hat{\mathbf{z}}_{2}\right\} \quad$ - PMU plane coordinate system (PPS)

$\left\{\hat{\mathbf{x}}_{p}, \hat{\mathbf{y}}_{p}, \hat{\mathbf{z}}_{p}\right\}$ - PMU coordinate system (PUS)

WCS is attached to the five-bar linkage base, with $\hat{\mathbf{y}}_{w}$ lying along the $\operatorname{link} l_{1}$ and $\hat{\mathbf{z}}_{w}$ normal to the five-bar plane. FES is attached to the EE of the five-bar linkage, with $\hat{\mathbf{z}}_{t}$ parallel to $\hat{\mathbf{z}}_{w}$ and $\hat{\mathbf{x}}_{t}$ along the direction of the $\operatorname{link} l_{6}$. SAS is defined such that $\hat{\mathbf{z}}_{d}$ along the direction of the snake arm, and $\hat{\mathbf{y}}_{d}=\hat{\mathbf{z}}_{d} \times \hat{\mathbf{x}}_{t}$. BDS is obtained by rotating SAS about $\hat{\mathbf{z}}_{d}$ with an angle $q_{4}$. Rotating BDS about $\hat{\mathbf{z}}_{b}$ further gives SPS, so that $\hat{\mathbf{x}}_{1}$ lies in the SLU bending plane. EDS is attached to the SLU end disk, and is obtained from SPS by a simple rotation about $\hat{\mathbf{y}}_{1}$ so that $\hat{\mathbf{z}}_{1}$ becomes the backbone tangent at the end disk. GCS is defined in the same way as BDS, so that $\hat{\mathbf{x}}_{g}$ pointing from the central hole to the outer hole. PPS is defined like SPS so that $\hat{\mathbf{x}}_{2}$ lies in the bending plane of the PMU. Finally, PUS is the coordinate system attached to the PMU platform, and it is obtained by rotating PPS about axis $\hat{\mathbf{y}}_{2}$ so that $\hat{\mathbf{z}}_{p}$ is normal to the parallel platform disk.

Upon defining all coordinate systems, the following notations used in this paper are also defined below: $\{a\}$ - A right handed coordinate system named $a$. $q_{1}, q_{2}$ - Rotary actuation angles of the five-bar linkage. $\alpha_{1}, \alpha_{2}$ - Auxiliary angles for solving linkage kinematics. $l_{1} \ldots l_{6}$ - Link dimensions of the five bar.

$\mathrm{P}_{\mathrm{F}}$ - The supporting point inside the laryngoscope.

$\mathrm{P}_{\mathrm{U}} \mathrm{P}_{\mathrm{D}} \mathrm{P}_{\mathrm{L}} \mathrm{P}_{\mathrm{P}}$ - Origins of frames $\{t\}\{d\}\{e\}\{p\}$.

$L_{\mathrm{a}}$ - The length of the snake arm .(DDU holder, Fig. 2)

$q_{3}, q_{4}$ - Actuation variables of the Z- $\theta$ stage where $q_{3}$ is for translation and $q_{4}$ for rotation.

$i$ - Index of the secondary backbones $i=1,2,3$.

$s$ - Arc-length parameter of the primary backbone for SLU.

$\mathrm{s}=0$ at the base disk and $s=L$ at the end disk.

$L$ - Length of the SLU primary backbone.
$L_{i}$ - Length of the SLU $i^{\text {th }}$ backbone from base to end disk. $\theta(s)$ - Angle of the primary backbone tangent in the SLU bending plane. $\theta_{L}$ and $\theta_{0}$ designate $\theta(s=L)$ and $\theta(s=0)$.

$\delta$ - The snake bending plane angle.

$L$ - Length of the primary backbone of the PMU.

$s$-Arc-length parameter of the primary backbone for PMU. $s^{\prime}=0$ at the gripper end disk and $s^{\prime}=L^{\prime}$ at the platform disk. $\phi\left(s^{\prime}\right)$ - Angle of the primary backbone tangent in the PMU bending plane. $\phi_{L^{\prime}}$ and $\phi_{0}$ denote $\phi\left(s^{\prime}=L^{\prime}\right)$ and $\phi\left(s^{\prime}=0\right)$. $\psi$ - The PMU bending plane angle.

$\mathbf{p}_{\mathbf{A}}^{c}$ - Position vector of point A expressed in frame $\{c\}$.

${ }^{a} \mathbf{R}_{b}$ - Rotation matrix from frame $\{a\}$ to $\{b\}$.

$\operatorname{Rot}\left(\hat{\mathbf{z}}_{a}, \alpha\right)$ - Rotation matrix about axis $\hat{\mathbf{z}}_{a}$ with angle $\alpha$.

$\dot{\mathbf{x}}$ - Time derivative of variable $\mathbf{x}$.

$\mathbf{J}_{\text {unit }}^{a}$ - Jacobian matrix of a certain unit in frame $\{a\}$.

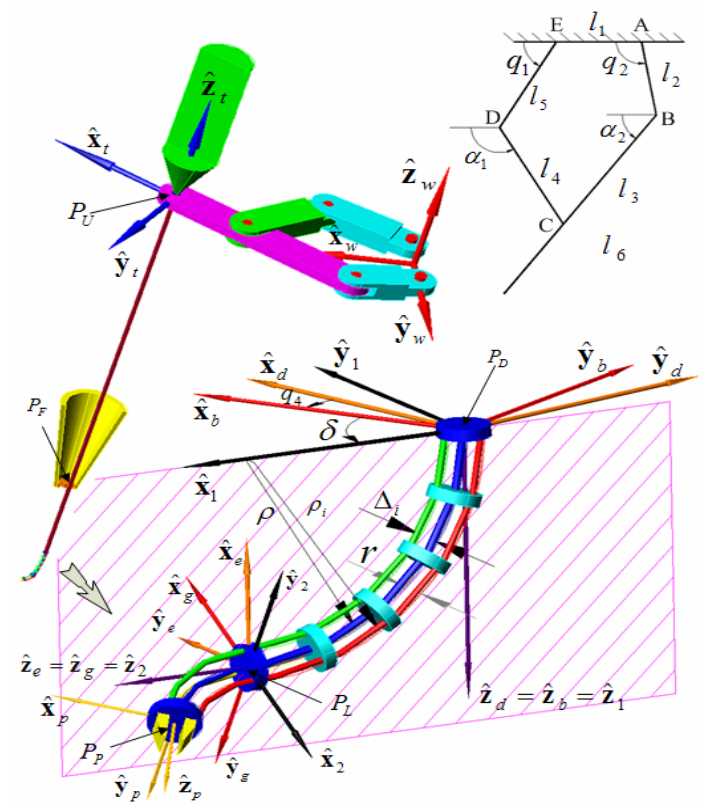

Figure 4. Kinematic nomenclature for one branch of the two-armed robot

\section{B. Kinematic Relations}

1) Five-bar Linkage: The five-bar linkage has two inputs, the actuated angles of rotation $q_{1}$ and $q_{2}$, and two outputs, the position of the $\operatorname{EE} x_{\mathbf{P}_{\mathbf{U}}}$ and $y_{\mathbf{P}_{\mathbf{U}}}$. The rotation matrix from $\{w\}$ to $\{t\}$ is given in Eq. (2). Points $\mathrm{D}$ and $\mathrm{B}$ are given by Eqs $(3,4)$. Point $\mathrm{C}$ can be derived as in Eq. (5). The two angles $\alpha_{1}$ and $\alpha_{2}$ in Eq. (5) can be solved in terms of $q_{1}$ and $q_{2}$, thus $\alpha_{1}=\alpha_{1}\left(q_{1}, q_{2}\right)$ and $\alpha_{2}=\alpha_{2}\left(q_{1}, q_{2}\right)$. Therefore, the position vector of $\mathrm{P}_{\mathrm{U}}$ is as in Eq. (6).

$$
\begin{gathered}
{ }^{w} \mathbf{R}_{t}=\operatorname{Rot}\left(\hat{\mathbf{z}}_{w}, \alpha_{2}-\pi / 2\right) \\
\mathbf{p}_{\mathbf{D}}^{w}=\mathbf{l}_{5}\left(q_{1}\right)=\left[l_{5} \sin \left(q_{1}\right), l_{5} \cos \left(q_{1}\right)-l_{1} / 2,0\right]^{T} \\
\mathbf{p}_{\mathbf{B}}^{w}=\mathbf{l}_{2}\left(q_{2}\right)=\left[l_{2} \sin \left(q_{2}\right), l_{2} \cos \left(q_{2}\right)+l_{1} / 2,0\right]^{T} \\
\mathbf{p}_{\mathbf{C}}^{w}=\mathbf{p}_{\mathbf{D}}^{w}+\mathbf{l}_{4}\left(\alpha_{1}\right)=\mathbf{p}_{\mathbf{B}}^{w}+\mathbf{l}_{3}\left(\alpha_{2}\right) \\
\mathbf{p}_{\mathbf{P}_{\mathbf{U}}}^{w}=\mathbf{p}_{\mathbf{B}}^{w}+\mathbf{l}_{6}\left(\alpha_{2}\right)
\end{gathered}
$$


2) Z- $\boldsymbol{\theta}$ Stage: The $Z-\theta$ stage provides the snake arm two DoF, one translation along the arm axis, and one rotation about the same axis. The rotation matrix from $\{t\}$ to $\{b\}$ is where ${ }^{d} \mathbf{R}_{b}=\operatorname{Rot}\left(\hat{\mathbf{z}}_{d}, q_{4}\right)$ and where $\hat{\mathbf{x}}_{d}, \hat{\mathbf{y}}_{d}, \hat{\mathbf{z}}_{d}$ are given in Eq. (7). Point $\mathrm{P}_{\mathrm{D}}$ in $\{t\}$ is given by Eq. (8)

$$
\begin{gathered}
\hat{\mathbf{z}}_{d}=\frac{\mathbf{p}_{\mathbf{P}_{\mathbf{F}}}^{t}-\mathbf{p}_{\mathbf{P}_{\mathbf{U}}}^{t}}{\left|\mathbf{p}_{\mathbf{P}_{\mathbf{F}}}^{t}-\mathbf{p}_{\mathbf{P}_{\mathbf{U}}}^{t}\right|}, \hat{\mathbf{y}}_{d}=\hat{\mathbf{z}}_{d} \times \hat{\mathbf{x}}_{t}, \hat{\mathbf{x}}_{d}=\hat{\mathbf{y}}_{d} \times \hat{\mathbf{z}}_{d} \\
{ }^{t} \mathbf{R}_{b}=\left[\hat{\mathbf{x}}_{d} \hat{\mathbf{y}}_{d} \hat{\mathbf{z}}_{d}\right]^{d} \mathbf{R}_{b} \\
\mathbf{p}_{\mathbf{P}_{\mathbf{D}}}^{t}=\left(L_{a}+q_{3}\right) \hat{\mathbf{z}}_{d}
\end{gathered}
$$

3) SLU: The direct kinematics of the SLU is expressed in $\{d\}$. For convenience we describe the kinematics of the SLU in its 2-dimensional configuration space. This space is parameterized by two angles $\theta_{L}$ and $\delta$. The results are given in Eqs. (10) and (11). For detailed derivations, please refer to previous papers $[20,21]$.

$$
\begin{gathered}
\mathbf{p}_{\mathbf{P}_{\mathbf{L}}}^{b}={ }^{b} \mathbf{R}_{1}\left[\int_{0}^{L} \cos (\theta(s)) d s, 0, \int_{0}^{L} \sin (\theta(s)) d s\right]^{T} \\
{ }^{b} \mathbf{R}_{g}={ }^{b} \mathbf{R}_{1}{ }^{1} \mathbf{R}_{e}{ }^{e} \mathbf{R}_{g}
\end{gathered}
$$

4) PMU: Since in this paper we solve the case where the PMU is embodied by a second SLU. The kinematics of this simplified PMU can be solved similarly as for the first SLU. Two variables, $\phi_{L^{\prime}}$ and $\psi$ are selected as the configuration space parameters for this snake-like unit. The kinematics of this unit is similar to the SLU with the correct change of variables. Point $\mathrm{P}_{\mathrm{P}}$ and the orientation of the gripper are given in Eqs. (12) and (13). ${ }^{g} \mathbf{R}_{2}$ is a rotation matrix of $(-\psi)$ about $\hat{\mathbf{z}}_{g} ;{ }^{2} \mathbf{R}_{p}$ is a rotation matrix of $\left(\phi_{0}-\phi_{L^{\prime}}\right)$ about $\hat{\mathbf{y}}_{2}$.

$$
\begin{gathered}
\mathbf{p}_{\mathbf{P}_{\mathbf{P}}}^{g}={ }^{g} \mathbf{R}_{2}\left[\int_{0}^{L^{\prime}} \cos \left(\phi\left(s^{\prime}\right)\right) d s^{\prime}, 0, \int_{0}^{L^{\prime}} \sin \left(\phi\left(s^{\prime}\right)\right) d s^{\prime}\right]^{T} \\
{ }^{g} \mathbf{R}_{p}={ }^{g} \mathbf{R}_{2}{ }^{2} \mathbf{R}_{p}
\end{gathered}
$$

\section{Jacobian Formulation}

The five bar mechanism: A general method to find the Jacobian matrix for a planar five-bar linkage is presented herein. The resultant force $\mathbf{f}_{e}$ and moment $m_{e}$ applied by the EE of the five-bar are resolved into actuator torques and then by using the virtual work principle it is easy to derive the instantaneous kinematics Jacobian.

$$
\begin{gathered}
\mathbf{f}_{e}=f_{1} \hat{\mathbf{e}}_{1}+f_{2} \hat{\mathbf{e}}_{2}+f_{3} \hat{\mathbf{e}}_{3} \\
m_{e}=\left(\mathbf{p}_{1} \times f_{1} \hat{\mathbf{e}}_{1}+\mathbf{p}_{2} \times f_{2} \hat{\mathbf{e}}_{2}+\mathbf{p}_{2} \times f_{3} \hat{\mathbf{e}}_{3}\right) \cdot \hat{\mathbf{z}}_{e}
\end{gathered}
$$

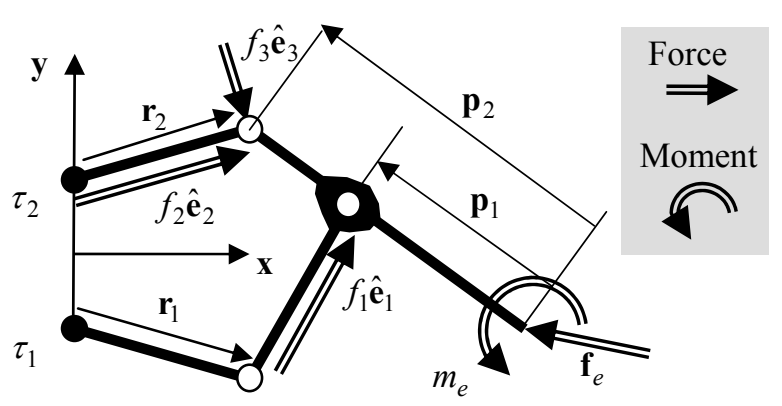

Figure 5. Static analysis model of the five-bar linkage where $\hat{\mathbf{z}}_{e}=[0,0,1]^{T}$. The matrix form of Eqs. (14) (15) is

$$
\mathbf{w}_{e}=\underbrace{\left[\begin{array}{ccc}
\hat{\mathbf{e}}_{1} & \hat{\mathbf{e}}_{2} & \hat{\mathbf{e}}_{3} \\
\left(\mathbf{p}_{1} \times \hat{\mathbf{e}}_{1}\right)^{T} \hat{\mathbf{z}}_{e} & \left(\mathbf{p}_{2} \times \hat{\mathbf{e}}_{2}\right)^{T} \hat{\mathbf{z}}_{e} & \left(\mathbf{p}_{2} \times \hat{\mathbf{e}}_{3}\right)^{T} \hat{\mathbf{z}}_{e}
\end{array}\right] \mathbf{f}}_{\mathbf{J}_{p}^{T}}
$$

where, $\quad \mathbf{w}_{e}=\left[\mathbf{f}_{e}^{T}, m_{e}\right]^{T}$ and $\mathbf{f}=\left[f_{1}, f_{2}, f_{3}\right]^{T}$. Eq. (17) provides the joint torques where $\boldsymbol{\tau}=\left[\tau_{1}, \tau_{2}\right]^{T}$ are the actuator torques. For parallel robots the statics is given by $\mathbf{J}^{T} \boldsymbol{\tau}=\mathbf{w}_{e},[24,25]$. Using this definition, one obtains the Jacobian as $\mathbf{J}_{5 b a r}^{w}$ in Eq. (18).

$$
\begin{gathered}
\boldsymbol{\tau}=\underbrace{\left[\begin{array}{ccc}
\left(\mathbf{r}_{1} \times \hat{\mathbf{e}}_{1}\right) \cdot \hat{\mathbf{z}}_{e} & 0 & 0 \\
0 & 0 & \left(\mathbf{r}_{2} \times \hat{\mathbf{e}}_{3}\right) \cdot \hat{\mathbf{z}}_{e}
\end{array}\right]}_{\mathbf{J}_{S}^{T}} \mathbf{f} \\
\boldsymbol{\tau}=\underbrace{\mathbf{J}_{S}^{T} \mathbf{J}_{P}^{-T}}_{\mathbf{J}_{5 b a r}^{w}} \mathbf{w}_{e}
\end{gathered}
$$

The Z- $\boldsymbol{\theta}$ stage: The Z- $\theta$ stage also has two DoF. The linear and angular velocities of $Z-\theta$ stage in FES are derived as

$$
\mathbf{v}_{z \theta}^{t}=\dot{q}_{3} \hat{\mathbf{z}}_{d} \quad \boldsymbol{\omega}_{z \theta}^{t}=\dot{q}_{4} \hat{\mathbf{z}}_{d}
$$

To transform the velocities into WCS, one obtains

$$
\mathbf{v}_{z \theta}^{w}={ }^{w} \mathbf{R}_{t}\left(\dot{q}_{3} \hat{\mathbf{z}}_{d}\right) \quad \boldsymbol{\omega}_{z \theta}^{w}={ }^{w} \mathbf{R}_{t}\left(\dot{q}_{4} \hat{\mathbf{z}}_{d}\right)
$$

The generalized twist of Z- $\theta$ stage with respect to FES expressed in WCS is obtained.

$$
\dot{\mathbf{x}}_{z \theta}^{w}=\left(\mathbf{J}_{z \theta}^{w}\right)_{6 \times 2}\left[\begin{array}{ll}
\dot{q}_{3} & \dot{q}_{4}
\end{array}\right]^{T}
$$

Snake-like unit: The twist of the SLU end disk is obtained by differentiating Eqs. (10) and (11) with respect to $\theta_{L}$ and $\delta$, Eqs. $(22,23)$ where $\mathbf{p}_{\mathrm{P}_{\mathrm{L}}}^{1}={ }^{1} \mathbf{R}_{b} \mathbf{p}_{\mathrm{P}_{\mathrm{L}}}^{b}$.

$$
\begin{gathered}
\mathbf{v}_{S L U}^{b}={ }^{b} \mathbf{R}_{1}\left(\dot{\mathbf{p}}_{\mathbf{P}_{\mathbf{L}}}^{1}+(-\dot{\delta}) \hat{\mathbf{z}}_{1} \times \mathbf{p}_{\mathbf{P}_{\mathbf{L}}}^{1}\right) \\
\boldsymbol{\omega}_{S L U}^{b}=-\dot{\delta} \dot{\mathbf{z}}_{1}-\dot{\theta}_{L} \hat{\mathbf{y}}_{1}+\dot{\tilde{\delta}}_{e}
\end{gathered}
$$

Transforming the velocities into WCS, one obtains

$$
\mathbf{v}_{S L U}^{w}=\boldsymbol{\omega}_{z \theta}^{w} \times\left({ }^{w} \mathbf{R}_{b} \mathbf{p}_{\mathrm{P}_{\mathrm{L}}}^{b}\right)+{ }^{w} \mathbf{R}_{b} \mathbf{v}_{S L U}^{b}
$$




$$
\boldsymbol{\omega}_{S L U}^{w}={ }^{w} \mathbf{R}_{b}\left(-\dot{\delta}_{1}-\dot{\theta}_{L} \hat{\mathbf{y}}_{1}+\dot{\delta}_{e}\right)
$$

The generalized twist of the SLU stage with respect to SAS expressed in WCS is obtained

$$
\dot{\mathbf{x}}_{S L U}^{w}=\left(\mathbf{J}_{S L U}^{w}\right)_{6 \times 3}\left[\begin{array}{lll}
\dot{q}_{4} & \dot{\theta}_{L} & \dot{\delta}
\end{array}\right]^{T}
$$

The PMU: Similarly, for the PMU, the linear and angular velocities of the parallel platform disk with respect to GCS expressed in WCS are derived as

$$
\mathbf{v}_{P M U}^{w}=\left(\boldsymbol{\omega}_{z \theta}^{w}+\boldsymbol{\omega}_{S L U}^{w}\right) \times\left({ }^{w} \mathbf{R}_{g} \mathbf{p}_{\mathrm{P}_{\mathrm{P}}}^{g}\right)+{ }^{w} \mathbf{R}_{g} \mathbf{v}_{P M U}^{g}
$$

$$
\text { where } \begin{aligned}
& \mathbf{v}_{P M U}^{g}={ }^{g} \mathbf{R}_{2}\left(\dot{\mathbf{p}}_{\mathbf{P}_{\mathbf{P}}}^{2}+(-\dot{\psi}) \hat{\mathbf{z}}_{2} \times \mathbf{p}_{\mathbf{P}_{\mathbf{P}}}^{2}\right) \\
& \boldsymbol{\omega}_{P M U}^{w}={ }^{w} \mathbf{R}_{g}\left(-\dot{\psi} \hat{\mathbf{z}}_{2}-\dot{\phi}_{L}, \hat{\mathbf{y}}_{2}+\dot{\psi} \hat{\mathbf{z}}_{p}\right)
\end{aligned}
$$

The generalized twist of the PMU is obtained

$$
\dot{\mathbf{x}}_{P M U}^{w}=\left(\mathbf{J}_{P M U}^{w}\right)_{6 \times 5}\left[\begin{array}{lllll}
\dot{q}_{4} & \dot{\theta}_{L} & \dot{\delta} & \dot{\phi}_{L^{\prime}} & \dot{\psi}
\end{array}\right]^{T}
$$

Combined Jacobian: The Jacobian matrix for one branch of the two-armed slave robot of Fig. 2 is as in Eq. (30).

$$
\begin{gathered}
\dot{\mathbf{x}}_{\text {system }}^{w}=\dot{\mathbf{x}}_{t}^{w}+\dot{\mathbf{x}}_{z \theta}^{w}+\dot{\mathbf{x}}_{S L U}^{w}+\dot{\mathbf{x}}_{P M U}^{w}= \\
\left(\mathbf{J}_{\text {total }}^{w}\right)_{6 \times 8}\left[\begin{array}{llllllll}
\dot{q}_{1} & \dot{q}_{2} & \dot{q}_{3} & \dot{q}_{4} & \dot{\theta}_{L} & \dot{\delta} & \dot{\phi}_{L^{\prime}} & \dot{\psi}
\end{array}\right]^{T}
\end{gathered}
$$

\section{SUTURING TASK}

Figure 6 shows the proposed mode of operation for suturing in confined spaces. The DDU holder in Fig. 2 is rotated by the Z- $\theta$ stage and the SLU is used to convert this rotation to rotate about the axis of the gripper. We use this task in our kinematic simulations to demonstrate the capability of our slave robot to perform this task.
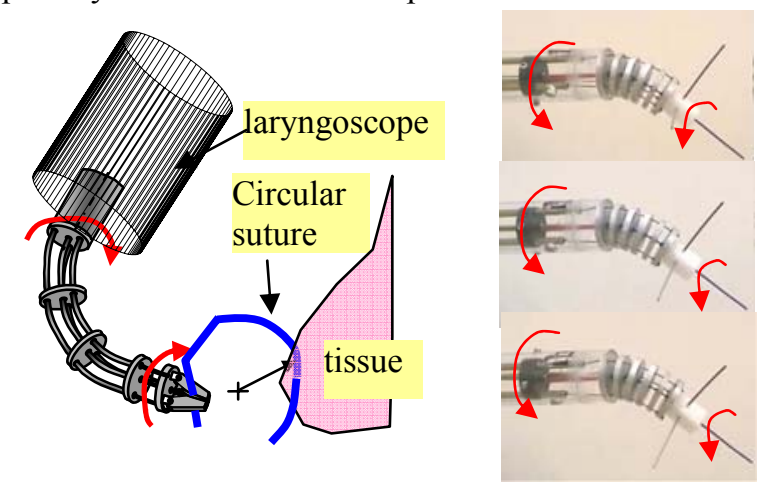

Figure 6. Suituring in confined spaces using the rotation about the primary backbone mode of operation [22], [21]

\section{OPTIMIZATION AND SIMULATION}

\section{A. Optimization Function}

We use redundancy resolution to solve for the inverse kinematics to satisfy the suturing task in Fig. 6. The Hamiltonian of the constrained minimization problem is:

$$
\underset{\Delta \mathbf{q}}{\operatorname{Min}} \mathrm{z}^{*}=\frac{1}{2} \Delta \mathbf{q}^{T} \mathbf{W} \Delta \mathbf{q}+\boldsymbol{\eta}^{T}(\Delta \mathbf{x}-\mathbf{J} \Delta \mathbf{q})
$$

where $\mathbf{J}=\mathbf{J}_{\text {total }}^{w}, \Delta \mathbf{q}$ and $\Delta \mathbf{x}$ are $8 \times 1$ and $6 \times 1$ vectors respectively, denoting the input and output joint variations, $\boldsymbol{\eta}$ is a $6 \times 1$ Lagrange multiplier ranges, and $\mathbf{W}$ is a diagonal weight matrix. The solution is given by:

$$
\Delta \mathbf{q}=\mathbf{W}^{-1} \mathbf{J}^{T}\left(\mathbf{J W}^{-1} \mathbf{J}^{T}\right)^{-1} \Delta \mathbf{x}
$$

The weight matrix $\mathbf{W}$ is used to minimize the joint velocities (and as a result the motions) of the DDU holder inside the laryngoscope. A more general methodology was presented by [21].

\section{B. Inputs Optimization}

The robotic system presented in this paper is redundant in terms of inputs joints; therefore, an approach to reasonably allocate the range of motion for each joint is significant. With the same optimization function given in Eq. (31), two attempts are made to minimize the eight input joint ranges when the gripper performs the suturing task of Fig. 6. In the first attempt, the weight matrix $\mathbf{W}_{1}$ is an identity matrix. In the second attempt, $\mathbf{W}_{2}=\operatorname{diag}[1000$

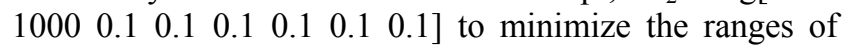
inputs $\mathrm{q}_{1}$ and $\mathrm{q}_{2}$. The path of a throat suturing operation is a half circle, which follows the shape of the suture. In the simulation, the starting pose for the inputs is [1.4522, $2.4707,50,0, \pi / 2,-\pi / 3,0,-\pi / 3]^{\mathrm{T}}$. The radius of the path is $6 \mathrm{~mm}$, determined by the practical dimensions of a suture. The EE moves at a velocity of constant magnitude $0.5 \mathrm{~mm} / \mathrm{s}$. Comparative plots under different weight matrices for all input joints are given in Fig. 7, in which $\left|\mathrm{q}_{i} / \mathrm{q}_{\text {imax }}\right|$ is plotted instead of $\mathrm{q}_{\mathrm{i}}$ for each joint. The comparison shows that by specifying the weight matrix $\mathbf{W}_{2}$, the ranges of motion of inputs $\mathrm{q}_{1}$ and $\mathrm{q}_{2}$ are reduced, while some other inputs are increased to different extents. Overall all joints remain within their limits.

\section{CONCLUSION}

This paper presented the mechanical architecture of a novel two-armed slave manipulator developed for MIS of the throat. Each 8 DoF arm is a hybrid robot consisting of a parallel robot, a serial kinematic chain, and a snake-like unit used for dexterity enhancement. The paper develops the kinematics of each arm by defining an augmented vector consisting of the joint variables of the serial/parallel structure and the configuration variables of the snake-like unit. Redundancy resolution using a weighted least squares formulation is used to demonstrate the capabilities of this robot to perform suturing in very confined spaces. The mode of operation chosen for suturing uses the capability of the snake-like unit to transform rotation along its backbone. As a result minimization of tissue tearing is achieved. The results of our simulation show that the robot is capable of performing this demanding task. 

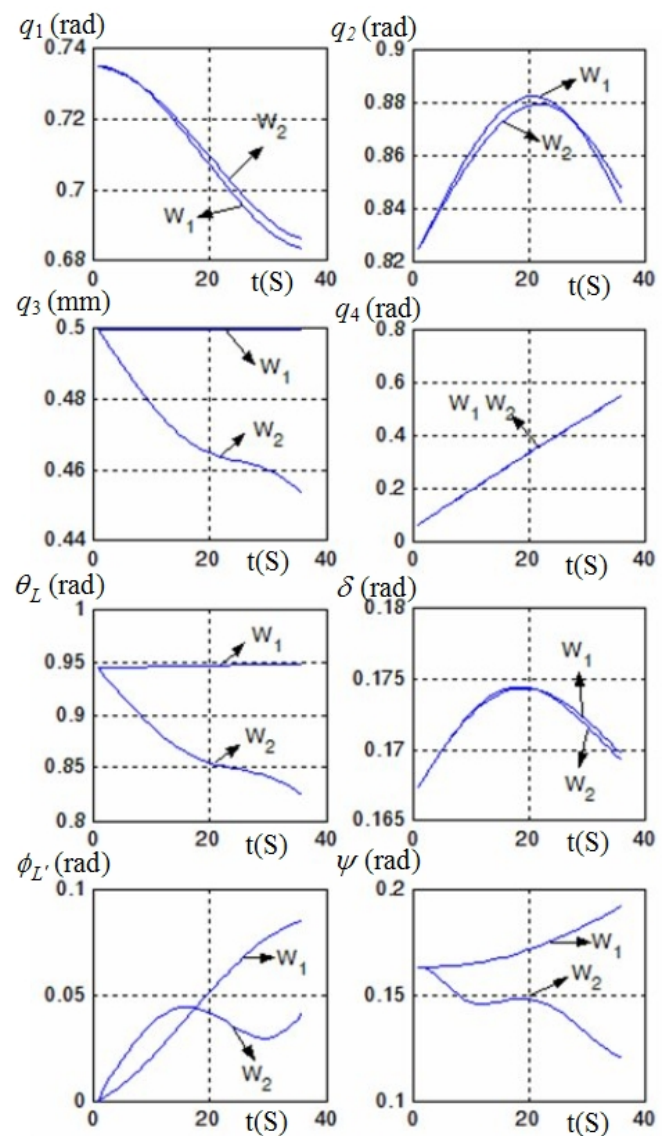

Figure 7. Simulation results of the suturing task in Fig. 6. The figure shows the joint ranges $\left|\mathrm{q}_{\mathrm{i}} / \mathrm{q}_{\max }\right|$

\section{ACKNOWLEDGMENT}

This work was partially funded by the National Science Foundation (NSF) under Engineering Research Center grant \#EEC9731478, NSF grant \#IIS9801684, and by the National Institutes of Health (NIH) under grant \#R21 B004457-01 and by Columbia University and Johns Hopkins University internal funds.

\section{REFERENCES}

[1] Charles, S., "Dexterity Enhancement for Surgery," R. H. Taylor, S. Lavallee, G. C. Burdea, and R. Mosges, Eds. Cambridge, MA: MIT Press, 1996, pp. 467-472.

[2] Minor, M. and Mukherejee, R., "A Dexterous Manipulator for Minimally Invasive Surgery," IEEE International Conference on Robotics and Automation, pp. 2057-2064, 1999.

[3] Yamashita, H., Kim, D., Hata, N., and Dohi, T., "Multi-Slider Linkage Mechanism for Endoscopic Forceps Manipulator," IEEE International Conference on Intelligent Robots and Systems (IROS), pp. 2577-2582, 2003.

[4] Reboulet, C. and Durand-Leguay, S., "Optimal design of redundant parallel mechanism for endoscopic surgery," IEEE International Conference on Intelligent Robots and Systems, pp. 1432-1437, 1999.

[5] Piers, J., D. Reynaerts, and Brussel, H. V., "Design of Miniature Parallel Manipulators for Integration in a Self-propelling Endoscope," Sensors and Actuators, vol. 85, pp. 409-417, 2000.

[6] Merlet, J.-P., "Optimal design for the micro parallel robot MIPS," IEEE International Conference on Robotics and Automation, pp. 1149-1154, 2002

[7] Guthart, G. and Salisbury, K., "The Intuitive ${ }^{\mathrm{TM}}$ Telesurgery System: Overview and Application," IEEE International Conference on Robotics and Automation, pp. 618-621, 2000.
[8] Madhani, A. J., Niemeyer, G., and Salisbury, K., "The Black Falcon: A Teleoperated Surgical Instrument for Minimally Invasive Surgery," IEEE/RSJ International Conference on Intelligent Robots and Systems (IROS), pp. 936-944, 1998.

[9] Schenker, P. S., Barlow, E. C., Boswell, C. D., Das, H., Lee, S., Ohm, T. R., Paljug, E. D., Rodriguez, G., and Charles, S., "Development of a Telemanipulator for Dexterity Enhanced Microsurgery," 2nd Annual International Symposium on Medical Robotics and Computer Assisted Surgery (MRCAS), pp. 81-88, 1995.

[10] Harada, K., Tsubouchi, K., Fujie, M. G., and Chiba, T., "Micro Manipulators for Intrauterine Fetal Surgery in an Open MRI," IEEE International Conference on Robotics and Automation, pp. 504-509, 2005.

[11] Ikuta, K., Yamamoto, K., and Sasaki, K., "Development of Remote Microsurgery Robot and New Surgical Procedure for Deep and Narrow Space," IEEE International Conference on Robotics and Automation, pp. 1103-1108, 2003.

[12] Dario, P., Paggetti, C., Troisfontaine, N., Papa, E., Ciucci, T. Carrozza, M. C., and Marcacci, M., "A Miniature Steerable EndEffector for Application in an Integrated System for ComputerAssisted Arthroscopy," IEEE International Conference on Robotics and Automation, pp. 1573-1579, 1997.

[13] Piers, J., Reynaerts, D., Van Brussel, H., De Gersem, G., and Tang, H. T., "Design of an Advanced Tool Guiding System for Robotic Surgery," IEEE International Conference on Robotics and Automation, pp. 2651-2656, 2003.

[14] D'attansio, S., Tonet, O., Megali, G., Carrozza, M. C., and Dario, P., "A Semi-Automatic Handheld Mechatronic Endoscope with Collision-Avoidance Capabilities," IEEE International Conference on Robotics \& Aotomation, pp. 1586-1591, 2000.

[15] Dario, P., Carrozza, M. C., Pietrabissa, A., and Surgery, C. A., "Developmnet and In Vitro Testing of a Miniature Robotic System for Comuter-Assisted Clonoscopy," vol. 4, pp. 1-14, 1999.

[16] Reynaerts, D., Peirs, J., and Van Brussel, H., "Shape memory micro-actuation for a gastro-intesteinal intervention system," Sensors and Actuators, vol. 77, pp. 157-166, 1999.

[17] Taylor, R. and Stianovici, D., "Medical Robotics in ComputerIntegrated Surgery," IEEE Transactions on Robotics and Automation, vol. 19, pp. 765-781, 2003.

[18] Tendick, F., Sastry, S., Fearing, R., and Cohn, M., "Applications of Microelectromechanics in Minimally Invasive Surgery," IEEE/ASME Transactions on Mechatronics, vol. 3, pp. 34-42, 1998.

[19] Asai, D., Katopo, S., Arata, J., Warisawa, S. i., Mitsuishi, M. Morita, A., Sora, S., Kirino, T., and Mochizuki, R., "MicroNeurosurgical System in the Deep Surgical Field," MICCAI 2004 (7th International Conference on Medical Image Computing and Computer-Assisted Intervention), pp. 33-40, 2004.

[20] Simaan, N., "High Dexterity Snake-like Robotic Slaves for Minimally Invasive Telesurgery of the Upper Airway," MICCAI 2004 (7th International Conference on Medical Image Computing and Computer-Assisted Intervention), pp. 17-24, 2004.

[21] Kapoor, A., Simaan, N., and Taylor, R., "Suturing in Confined Spaces: Constrained Motion Control of a Hybrid 8-DoF Robot," International Conference on Advanced Robotics (IACR'2005), Seattle, USA, pp. 452-459, 2005.

[22] Simaan, N., "Snake-Like Units Using Flexible Backbones and Actuation Redundancy for Enhanced Miniaturization," IEEE International Conference on Robotics and Automation, Barcelona, Spain, pp. 3020-3028, 2005.

[23] Simaan, N. and Taylor, R., "A System for Macro-Micro Distal Dexterity Enhancement in Micro Surgery of the Eye - a report of invention," Johns Hopkins ERC-CISST 5/13/2004 2004.

[24] Simaan, N., "Analysis and Synthesis of Parallel Robots for Medical Applications," in Mechanical Engineering, vol. M.Sc. Haifa, Israel: Technion, 1999.

[25] Tsai, L.-W., Robot Analysis: The Mechanics of Serial and Parallel Manipulators: John-Wiley \& Sons, INC., 1999.

[26] Yoshikawa, T., Foundations of Robotics Analysis and Control: MIT Press, 1990. 